Artículo original

\title{
Años potenciales de vida perdidos por incidentes viales de motociclistas, Medellín, 2009- 2012: un análisis espacial por sitio de la ocurrencia
}

\author{
Sandra Porras ${ }^{1}$; Grisales Hugo ${ }^{2,}$, hugo.grisales@udea.edu.co
}

Profesional en gerencia de sistemas de información en salud, Universidad de Antioquia, Medellín, Colombia. PhD Epidemiología, profesor Facultad Nacional de Salud Pública, Universidad de Antioquia, Medellín, Colombia

Fecha de recepción marzo 30 de 2016 Fecha de correcciones agosto 15 de 2016 Fecha de aceptación septiembre 30 de 2016 Fecha de publicación enero 31 de 2017

\section{Resumen}

Introducción. La accidentalidad de motociclistas es una problemática de salud pública de Medellín, que afecta inequitativamente a la población. Objetivo. Describir los años potenciales de vida perdidos (APVP) por accidentes de motociclistas en Medellín entre 2009 y 2012. Métodos. Se realizó un estudio descriptivo con fuente de información secundaria ajustada con el método de Preston y Coale, y OPS de distribución proporcional; en el cálculo de los APVP se empleó como valor de referencia la proyección anual de la esperanza de vida al nacer de Colombia 2009-2014 y para el análisis geográfico se empleó el método cuantil de clasificación, la densidad de Kernel y la simbología por cantidades y atributos múltiples. Resultados. El 79,4\% de los muertos en incidentes viales fueron hombres, el 50\% tenía 40 años o menos ( $\mathrm{RQ}=35$ años); los peatones $(45,6 \%$ ) y los motociclistas $(31,7 \%)$ fueron los usuarios más vulnerables de la vía. Los motociclistas hombres entre los 15 y 29 años tuvieron la mayor pérdida de años potenciales de vida, al igual que los accidentados en los barrios Caribe, San Cristóbal, Guayabal y Castilla; la mayor frecuencia de motociclistas muertos se observó en tramos de la autopista norte, la autopista sur, la carrera 65, la avenida regional y la avenida guayabal. Conclusión. En Medellín los motociclistas jóvenes de sexo masculino presentan las tasas de APVP más altas del periodo, lo que implica mayores costos sociales y económicos para la ciudad. La accidentalidad fatal más alta de este grupo de interés se presenta en las vías principales de la ciudad aledañas al río Medellín.

Palabras clave: Accidentes de Tránsito, Motocicletas, APVP, Resultado Fatal, Tasa de mortalidad.

Copyright $\odot$ Facultad de Ciencias de la Salud de la Universidad Tecnológica de Pereira. 1995-2017. Todos los derechos reservados ${ }^{*}$
Potential years of life lost due to road incidents of motorcyclists, Medellin, 2009-2012: a spatial analysis of the occurrence site

Abstract

Introduction. Motorcycle accidents is a public health problem of Medellin, which affects unfairly the population. Objective. To describe the potential years of life lost (YPLL) motorcycle accident in Medellin between 2009 and 2012. Methods: A descriptive study was conducted with secondary information source adjusted with the Preston and Coale method; in the calculation of YPLL, the annual projection of life expectancy at birth of Colombia 2009-2014 was used as reference value, and for the geographic analysis the "Quantile" classification method, Kernel density and symbology amounts and multiple attributes were used. Results: $79.4 \%$ of those killed in road accidents were men, $50 \%$ were 40 years old or less; pedestrians (45.6\%) and motorcyclists (31.7\%) were the most vulnerable road users. Motorcyclists men between 15 and 29 years of age had the highest amount of potential years of life lost, in the same way, the road accidents in Caribe, San Cristóbal, Guayabal and Castilla neighborhoods; the increased frequency of motorcyclists killed was observed in sections of the highway north, the south highway, running 65 regional and Guayabal Avenue. Conclusion: In Medellin young male motorcyclists have YPLL rates highest in the period, which implies greater social and economic costs for the city. The highest fatal accident rate of this interest group is presented on the main roads of the city along the Medellin River. ${ }^{\circ}$.

Key words: Road accidents, motorcycles, YPLL, fatal outcome, mortality rate.

Copyright $\odot$ Facultad de Ciencias de la Salud de la Universidad Tecnológica de Pereira. 1995-2017. All rights reserved

\section{Introducción}

A nivel mundial el panorama epidemiológico ha cambiado ampliamente en las últimas décadas, donde los nuevos desafíos sanitarios se encuentran enmarcados más que todo por patologías no transmisibles, cuya complejidad en su atención requiere de cambios significativos en los sistemas de salud, para lo cual es indispensable la adopción de nuevas metodologías que permitan la medición e identificación de tendencias de dichos eventos de una manera más integral, mejorando la definición de prioridades en salud a nivel global, privilegiando los logros en calidad de vida y sobrevida [1]. De esta manera, el principal desafío para este siglo, en cuanto a salud pública se refiere, ha sido la asignación adecuada de los recursos disponibles, con el fin de mejorar el marco causal de morbi mortalidad a nivel mundial, y en especial, reducir la marcada brecha en salud de los países.

Actualmente, uno de los problemas relevantes en la salud pública mundial son los accidentes de tránsito, definidos como aquellas colisiones generadas al menos por un vehículo en movimiento en una vía pública o privada, que causa daños a personas y bienes involucrados [2] debido a que cada día, miles de personas pierden la vida, sufren traumatismos o quedan con secuelas graves a causa de estos, afectando a la población que se encuentra en los años más productivos de su vida, como lo son los adolescentes y adultos jóvenes, en su mayoría miembros de países de medianos y bajos ingresos [3], lo cual genera un impacto negativo en la sociedad, en la economía y en la calidad de vida de las personas [4].

Este tipo de incidentes se encuentra dentro de las principales causas de mortalidad evitable a nivel global, dejando alrededor de 1,2 millones de personas muertas y 50 millones de personas heridas anualmente [5], las proyecciones indican que estas cifras aumentarán en todo el mundo casi a un $65 \%$ y en los países de niveles económicos bajos y medios hasta un $80 \%$ para el año 2020, si no se intensifica las acciones y asume un compromiso encaminado a la prevención de estos hechos [6]. Diariamente a nivel mundial mueren alrededor de 16.000 personas a causa de todo tipo de traumatismo, predominando los generados por colisiones en la vía pública, donde los causados por el tránsito son el $25 \%$ de todas las muertes por traumatismo; esta problemática ya no constituye un patrimonio exclusivo de los países ricos, ahora, son las regiones de medio y bajos ingresos- África, Asia, Caribe y gran parte de América Latina- las que se ven más afectadas por estos eventos, representando el $80 \%$ de las muertes, siendo en su orden los motociclistas (23\%), peatones (22\%) y ciclistas (5\%) los usuarios más vulnerables de la vía pública, aunque esto 
último depende en gran parte de la región y la solvencia económica de los países; los hombres entre los 15 y 44 años son los más afectados por los accidentes de tránsito, presentándose como segunda causa de muerte mundial en los niños y jóvenes (5 a 14 y 15 a 29 años respectivamente) $[6,7]$.

El informe anual denominado Accidentes de Tránsito de la Comunidad Andina 2013 [8], registra para ese año 347.018 accidentes de tránsito en los países pertenecientes a esta comunidadBolivia, Colombia, Ecuador y Perú-, a nivel general se presenta una disminución leve $(0,2 \%)$ con respecto al año anterior, donde específicamente Ecuador es el país que presenta un incremento de $18,1 \%$ y Colombia una disminución de 3,9\% comparado al año anterior; los vehículos de automotor, dentro de los cuales clasifican las motocicletas, ocuparan el segundo lugar en los vehículos involucrados en los accidentes de tránsito con 311.908 vehículos (22,7\%), de los cuales 89.061 son de Colombia.

Colombia vive diversos cambios sociales, políticos y económicos que en gran parte han influido en el crecimiento del parque automotor en sus principales ciudades, en especial el de las motocicletas, las cuales se han convertido en uno de los bienes más importantes de los hogares colombianos [9]; sin embargo, el incremento de este medio de transporte ha supuesto una carga importante para la salud de los colombianos, que se manifiesta entre otras formas como traumatismos, lesiones y muertes a causa de los accidentes que pueden resultar de la interacción con dichos vehículos; en el año 2013 se atienden 48.042 casos de accidentes de transporte, de los cuales el $12,94 \%$ terminan en lesiones fatales, los más afectados son los hombres en edad económicamente activos, ocupando el primer lugar los usuarios de motocicletas [10].

Antioquia aparece como el departamento líder en la lista de afectados tanto por muertes como lesiones generadas por este tipo de accidentes presentando para el año 2010 una tasa de mortalidad de 14 por 100.000 hab. aproximadamente, afectando principalmente a los hombres de 20 a 34 años de edad siendo los motociclistas el modo de transporte más frecuente (36\% de la mortalidad), el Valle de Aburrá presenta una tasa de mortalidad por accidentes de transporte para el año en mención de 13 por 100.000 hab. [11].

La ciudad de Medellín es protagonista en estos hechos [10]; durante los últimos cinco años los motociclistas se han convertido en la población más vulnerable de las vías de este territorio, incluso por encima de los peatones, lastimando especialmente a la población masculina de los 20 a los 29 años de edad, en su mayoría de estrato bajo y medio [12]. Los vehículos tipo motos participan aproximadamente en el 55\% de los accidentes presentes en la ciudad; con relación al año 2010, en el año 2013 se registra un aumento del 52,3\% en el número de usuarios de moto muertos en accidentes, teniendo una representación significativa los accidentes tipo choque y caída de ocupante [13].

Durante años se han empleado distintos indicadores epidemiológicos que permiten la medición del impacto generado por el amplio grupo de enfermedades y lesiones que pueden desencadenar negativamente en la salud de la población, entre ellos están los años de vida potencialmente perdidos (APVP) que surge como un complemento a los indicadores tradicionales de mortalidad, el cual evalúa la importancia que tienen las diferentes causas de muerte desde su magnitud y temporalidad, y permite conocer el estado de salud de las comunidades, acoger estrategias en pro del mejoramiento y calidad de los niveles de atención e identificar las prioridades para la asignación de los recursos disponibles en el sector [10].

A partir de este panorama, la descripción de la mortalidad y de la pérdida de vida prematura de los usuarios de motocicletas (conductores y pasajeros), permite marcar pautas para el desarrollo de estrategias de intervención que promuevan el bienestar de esta población vulnerable con mayor equidad, lo cual promueve proyecciones económicas, sociales e institucionales sustentadas en indicadores que dan cuenta del fenómeno de manera específica.

De este modo, el principal objetivo de esta investigación fue describir los años potenciales de vida perdidos por accidentes de motociclistas en Medellín durante el periodo 2009-2012, con el fin de valorar el impacto que estos eventos tienen sobre el estado de salud de la población; para ello, se caracterizó la mortalidad por incidentes viales, especialmente de motociclistas, se determinó la pérdida de años potenciales de vida de estos según variables demográficas, $y$ se referenció geográficamente los APVP y el lugar de ocurrencia de los incidentes viales fatales de motociclistas en la ciudad durante el periodo en observación.

\section{Materiales y métodos}

En esta investigación, se adoptó la metodología general que se emplea para calcular los años de vida potenciales perdidos por una población, la cual se detalla en la literatura $[14,15]$. Se realizó un estudio descriptivo con fuente de información ajustada por el subregistro de mortalidad con el método de Preston y Coale [16], y la metodología de distribución proporcional propuesta por la Organización Panamericana de la Salud [17]; se tuvo en cuenta todos los registros oficiales de defunción (recolectados por el DANE) con causa básica de muerte relacionada con accidentes de tránsito de motociclistas (CIE-10: V20-V29) ocurridos en Medellín entre los años 2009 y 2012.

Se adoptó el método de los años potenciales de vida perdidos (APVP), donde el límite potencial fue la proyección anual de la esperanza de vida al nacer (EVN) de Colombia 2009-2014 (mujeres: 76,94 años; hombres: 70,83 años; total: 73,81 años), y aunque los anteriores valores no permiten una comparación internacional, se optó el uso de este valor límite de la edad para calcular los APVP porque se ajusta al perfil poblacional del país, lo cual permite hacer comparaciones entre zonas o regiones del mismo.

Para calcular los APVP se tuvo en cuenta grupos quinquenales de edad que permitieron que el cálculo fuera más preciso, por ello se empleó la marca de clase para comparar la EVN en cada grupo etario, obteniéndose los APVP de multiplicar el número de defunciones (por grupo de edad) con la diferencia entre la EVN y la marca de clase; este proceso se llevó a cabo para calcular los APVP por cada una de las características de interés. De igual modo, se ajustó por la estructura de la edad a través de la tasa de años potenciales de vida perdidos (IAPVP) (Tabla 1).

Tabla 1. Ilustración del cálculo de los APVP y IAPVP

\begin{tabular}{cccccccc}
\hline $\begin{array}{c}\text { Grupo } \\
\text { Edad (1) }\end{array}$ & Xi (2) & $\begin{array}{c}\text { Población } \\
(\mathbf{3})\end{array}$ & Muertes (4) & EVN (5) & EVN-Xi (6) & APVP (7) & IAPVP (8) \\
\hline Li-Ls & $(\mathrm{Li}+\mathrm{Ls}) / 2$ & - & $\begin{array}{c}\text { Casos } \\
\text { ajustados }\end{array}$ & $\begin{array}{c}\text { Límite } \\
\text { potencial }\end{array}$ & $(5-2)$ & $(4) \times(6)$ & $((7) /(3))^{*} 100.000$ \\
\hline
\end{tabular}


Para el análisis geográfico de las tasas de APVP por incidentes de motociclistas por barrio donde ocurrieron los hechos en el periodo de estudio se realizaron mapas temáticos por sexo y grupos de edad, teniendo en cuenta una simbología por cantidades y el método de clasificación estándar cuantil [18]; y para hacer la distribución espacial de los eventos fatales de motociclistas en la ciudad de Medellín por dirección de ocurrencia del hecho se tuvo presente un enfoque determinístico, dado a que cada entidad (defunción de motociclista) se le relacionó un identificador único que permitió distinguirlo de forma segura en el proceso de geocodificación [19].

Para ello, el primer paso consistió en la revisión de la variable disponible en el registro de defunción que refería la dirección donde ocurrieron los hechos, la cual se normalizó de acuerdo a cada uno de los componentes de la dirección, dejando de lado observaciones o descripciones textuales que pudieran presentar. Así mismo, se revisó la medida de la calidad de la geocodificación en términos de completitud, el cual fue del $84 \%$ aproximadamente; para la representación se empleó el método de la densidad de Kernel y la simbología por cantidades y atributos múltiples [20].

Para ubicar específicamente el número de eventos por dirección, fue necesario agrupar las muertes por localización y asociar dicho valor a cada una de las direcciones involucradas, lo cual permitió identificar la cantidad de defunciones de motociclistas por nomenclatura vial registrada, para ello, se realizó un mapa temático con simbología por atributos múltiples que permitió denotar las cantidades asociadas de forma gradual en cada poli línea de la malla vial correspondiente, categorizadas por anchos y colores diferentes de acuerdo al número de muertes. Para identificar el ranking de las vías con mayores muertes fue necesario hacer una revisión minuciosa de las direcciones que registraban alguna muerte, agrupándolas por nombre común de la vía (por lo general, para las avenidas y autopistas) y por calles o carreras; y para enseñar los tramos de las vías en los que la frecuencia de los accidentes mortales fue mayor, se hizo una selección por atributos en las cinco vías con mayor porcentaje de participación.

Para el análisis se hizo uso del software estadístico IBM SPSS $21^{\circ}$, de la hoja de cálculo Microsoft Excel y del geocodificador masivo de Medellín "MapGis" y de la plataforma de ArcGIS $10^{\circ}$ para la presentación de los mapas temáticos.

\section{Resultados}

En Medellín, en el periodo comprendido entre 2009 y 2012, se presentaron 1.222 defunciones a causa de los accidentes de tránsito, de las cuales el 79,4\% eran hombres, el 50\% de la población fallecida a raíz de dicha causa tenía 40 años o menos; los peatones $(45,6 \%$ ) y los motociclistas $(31,7 \%)$ fueron los usuarios más vulnerables de la vía.

En el año 2011 se observó la tasa de mortalidad por accidentes de motociclistas más alta del periodo (4,7 por cada cien mil habitantes) con una diferencia de casi dos unidades en la tasa con respecto a la más baja del cuatrienio. El 50\% de los motociclistas fallecidos tenían 27 años o menos; los motociclistas que fallecieron a causa de incidentes viales en Medellín del año 2009 a 2012 estaban iniciando la segunda década de la vida, notándose en todos los años evaluados una concentración considerable en los rangos de edad de 15 a 29 años y de 30 a 49 años (Tabla 2), correspondiendo esto a cerca del $96 \%$ de los usuarios de vehículo de dos llantas en la ciudad con desenlace fatal.

Tabla 2. Mortalidad por incidentes en motocicleta por grupos etarios y año. Medellín, 2009-2012a

\begin{tabular}{|c|c|c|c|c|c|c|c|c|c|c|c|c|c|c|c|}
\hline \multirow{2}{*}{ Año defunción } & \multicolumn{3}{|c|}{$1-14$} & \multicolumn{3}{|c|}{$15-29$} & \multicolumn{3}{|c|}{$30-49$} & \multicolumn{3}{|c|}{$50-64$} & \multicolumn{3}{|c|}{65 y más } \\
\hline & $\mathbf{n}$ & $\%^{\mathrm{a}}$ & Tasa $^{\mathrm{b}}$ & $\mathbf{n}$ & $\%^{\mathrm{a}}$ & Tasa $^{\mathrm{b}}$ & n & $\%^{\mathrm{a}}$ & Tasa $^{\mathrm{b}}$ & $\mathbf{n}$ & $\%^{\mathrm{a}}$ & Tasa $^{b}$ & $\mathbf{n}$ & $\%^{\mathrm{a}}$ & Tasa $^{\mathrm{b}}$ \\
\hline 2009 & 1 & 1,0 & 0,2 & 48 & 50,0 & 8,4 & 44 & 45,8 & 6,5 & 3 & 3,1 & 0,8 & 0 & 0,0 & 0,0 \\
\hline 2010 & 0 & 0,0 & 0,0 & 53 & 73,2 & 9,1 & 16 & 22,5 & 2,4 & 3 & 4,2 & 0,7 & 0 & 0,0 & 0,0 \\
\hline 2011 & 1 & 0,9 & 0,2 & 75 & 66,7 & 12,8 & 32 & 28,8 & 4,7 & 4 & 3,6 & 0,9 & 0 & 0,0 & 0,0 \\
\hline 2012 & 2 & 1,9 & 0,5 & 60 & 56,7 & 10,3 & 41 & 38,5 & 5,9 & 2 & 1,9 & 0,5 & 1 & 1,0 & 0,5 \\
\hline Total & 4 & 1,0 & 0,2 & 236 & 61,0 & 10,2 & 134 & 34,6 & 4,9 & 12 & 3,1 & 0,7 & 1 & 0,3 & 0,1 \\
\hline
\end{tabular}

a con respecto al total por año.

b Tasa por 100.000 hab.

Se observó marcado predominio de las defunciones en hombres motociclistas en una relación de 5 a 1 . El riesgo de presentarse un accidente mortal en motociclistas en la ciudad fue de 39,6 por cien mil; es decir, hubo aproximadamente 40 motociclistas víctimas fatales de accidentes por cada 100.000 motos que circularon en el área metropolitana en ese tiempo, observándose el mayor riesgo en el año 2009 (11,3 por cien mil). En promedio hubo 1 motociclista muerto por cada 1.212 motocicletas.

Durante el cuatrienio se presentó en total una pérdida de 17.478 años potenciales de vida a causa de los incidentes viales sufridos por los motociclistas en Medellín, con un promedio de 4.370 APVP por año lo que corresponde a una tasa de 751 APVP por cada 100.000 habitantes, siendo los años 2011 y 2012 los que aportaron cerca del $57 \%$ de los APVP en el periodo (Tabla 3 ).
Tabla 3. APVP por incidentes viales de motociclistas ocurridos en la ciudad de Medellín según sexo, 2009-2012

\begin{tabular}{ccccccc}
\hline \multirow{2}{*}{$\begin{array}{c}\text { Año } \\
\text { defunción }\end{array}$} & \multicolumn{2}{c}{ Total } & \multicolumn{2}{c}{ Hombres } & \multicolumn{2}{c}{ Mujeres } \\
APVP $^{\mathbf{a}}$ & $\begin{array}{c}\text { Tasa } \\
\text { APVP }^{\mathbf{b}}\end{array}$ & APVP $^{\mathbf{a}}$ & $\begin{array}{c}\text { Tasa } \\
\text { APVP }^{\mathbf{b}}\end{array}$ & APVP $^{\mathbf{a}}$ & $\begin{array}{c}\text { Tasa } \\
\text { APVP }^{\mathbf{b}}\end{array}$ \\
\hline 2009 & 4218 & 184,3 & 3228 & 299,9 & 811 & 66,9 \\
2010 & 3326 & 143,8 & 2352 & 216,2 & 863 & 70,4 \\
2011 & 5122 & 219,0 & 4105 & 373,2 & 782 & 63,1 \\
2012 & 4813 & 203,6 & 3901 & 350,9 & 688 & 55,0 \\
Total & 17478 & 751,4 & 13587 & 1242,0 & 3144 & 255,1 \\
\hline
\end{tabular}

${ }^{a}$ Con respecto al límite potencial de cada sexo y el general.

b Tasa mediana por 100.000 habitantes.

Con respecto al número total de APVP y a la tasa de los APVP fue notoria la participación del sexo masculino, casi 5 veces con respecto a las mujeres en todos los grupos etarios, presentándose la mayor concentración en el grupo de edad de los 15 a los 49 años. Tanto las tasas de los APVP de los hombres como las de las mujeres disminuyen paulatinamente a medida que aumenta la edad a partir de los 29 años (Figura 1). 


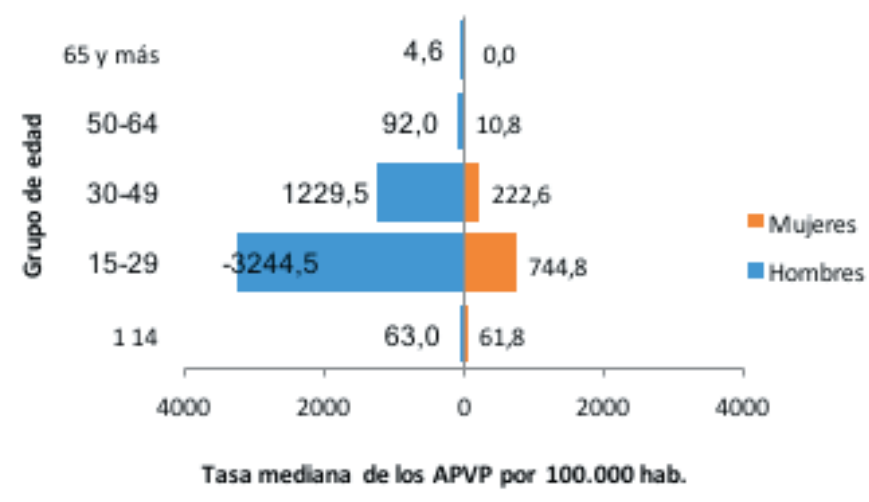

Figura 1. Distribución de la tasa de los APVP por incidentes viales de motociclistas en Medellín según grupo de edad y sexo, 2009-2012

En el periodo, la tasa más alta de los APVP por incidentes viales de motociclistas se presentó en la zona Noroccidental (188\%000 aproximadamente), seguida de la zona Centro Oriental y Suroccidental (aproximadamente $141 \% 000$ y $122 \% 000$ respectivamente); las cuales representaron el $60 \%$ de la pérdida de años de vida potenciales de motociclistas durante los años 2009-2012; específicamente en las comunas Castilla, La Candelaria, Guayabal, Aranjuez y Belén; donde se concentró cerca del 56\% de los APVP.

Las mayores tasas de los APVP por incidentes viales de motociclistas se presentaron en su orden en los barrios Caribe (35,5 APVP \%000), San Cristóbal (área urbana) (21,6 APVP \%000), Guayabal (21,2 APVP $\% 000)$, Castilla (17,0 APVP \%000), Campo Amor (16,6 APVP \%000), Cristo Rey (15,3 APVP \%000) y la Toscana (15,2 APVP \%000); en los cuales se concentró cerca del $20 \%$ de los años potenciales de vida perdidos por la causa en estudio. Cabe resaltar que los barrios con una tasa de APVP mayor a 5,4 por cada 100.000 hab. reunieron aproximadamente el 57\% del total de los APVP por incidentes viales de motociclistas en el periodo (40 barrios de 135 que refirieron la ocurrencia de algún accidente fatal de motociclista) (Figura 2).

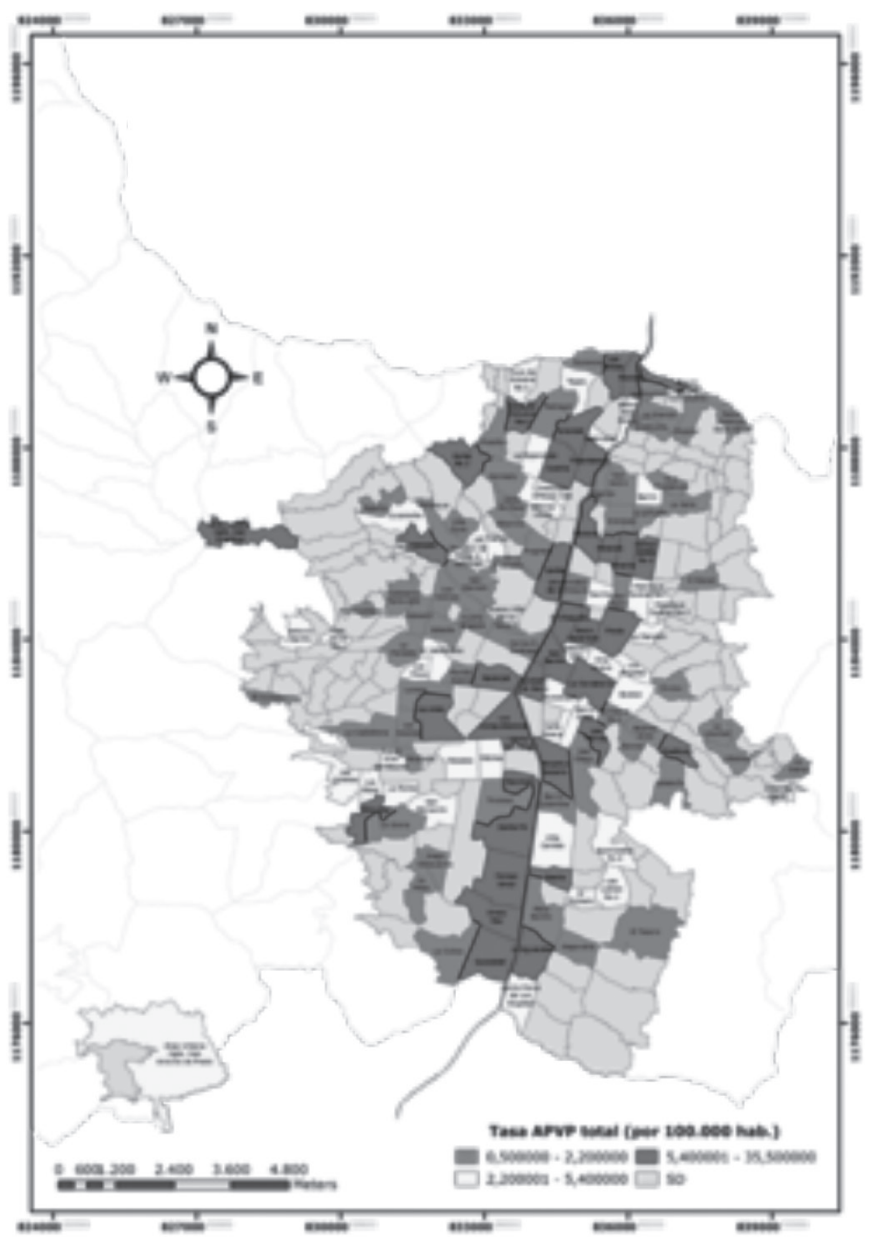

Figura 2. Tasa mediana de APVP por incidentes viales de motociclistas en los barrios de Medellín, 2009-2012

Los incidentes viales que generaron lesiones fatales en los usuarios de vehículo de motor de dos ruedas se presentaron con una mayor concentración en las zonas aledañas al río Medellín que la atraviesa de sur a norte, donde se encuentran importantes vías de comunicación y soportan una mayor circulación de vehículos (Figura 3). La densidad más alta de incidentes fatales se observó en las zonas que corresponden a las comunas Castilla, La Candelaria, Guayabal, Aranjuez, Belén y Laureles; las cuales fueron las primeras cinco comunas con una mayor tasa de mortalidad y tasa de APVP en el cuatrienio 


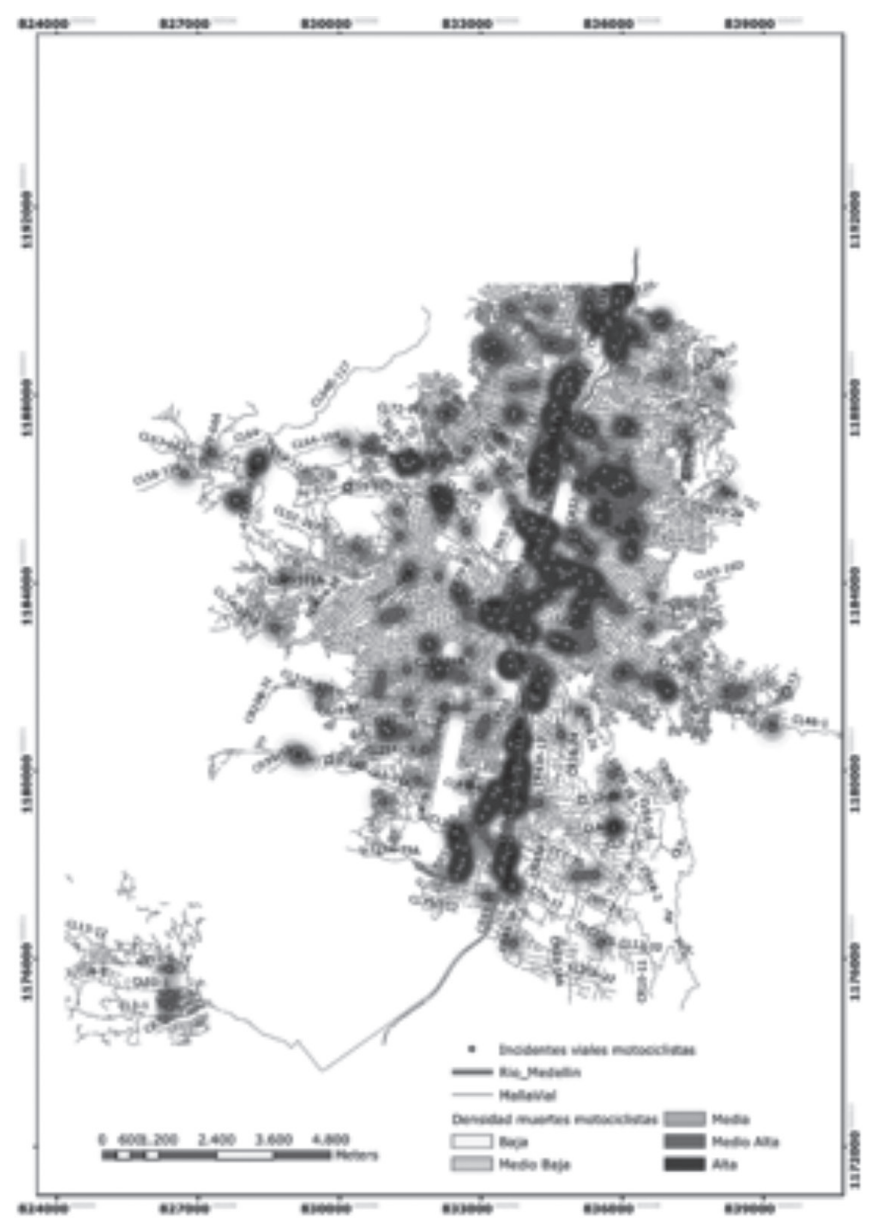

Figura 3. Densidad de muertes de motociclistas por incidentes viales en Medellín, 2009-2012

La mayor frecuencia de motociclistas muertos ocurrió en algunas de las principales vías de la ciudad, que se caracterizan por ser rápidas y admitir un gran volumen de tráfico; la autopista norte ocupó el primer lugar con el 5,7\% de las defunciones, siendo la $\mathrm{Cr} 64 \mathrm{C}$ (con la $78 \mathrm{~A}, 88 \mathrm{~A}, 93$ y $111 \mathrm{~A}$ ) el tramo con el número de muertos más alto (20 muertos); seguida de la autopista sur donde murió el 5,2\% de la población en estudio con una notoria participación de la $\mathrm{Cr} 63$ (8 muertos) y la Cr 50 (7 muertos); en tercer lugar la Cr 65 fue la vía en la que 18 motociclistas $(4,7 \%)$ perdieron la vida a causa de incidentes viales en el periodo. La avenida regional (en los tramos de la $\mathrm{Cr} 49$, Cr 62 y Cr 63) y la avenida guayabal (en la Cr 52) también fueron referentes geográficos importantes en la ocurrencia de los hechos que dieron lugar a la muerte de dichos usuarios de la vía $(4,4 \%$ y 3,4 \% de las muertes respectivamente) (Figura 4).

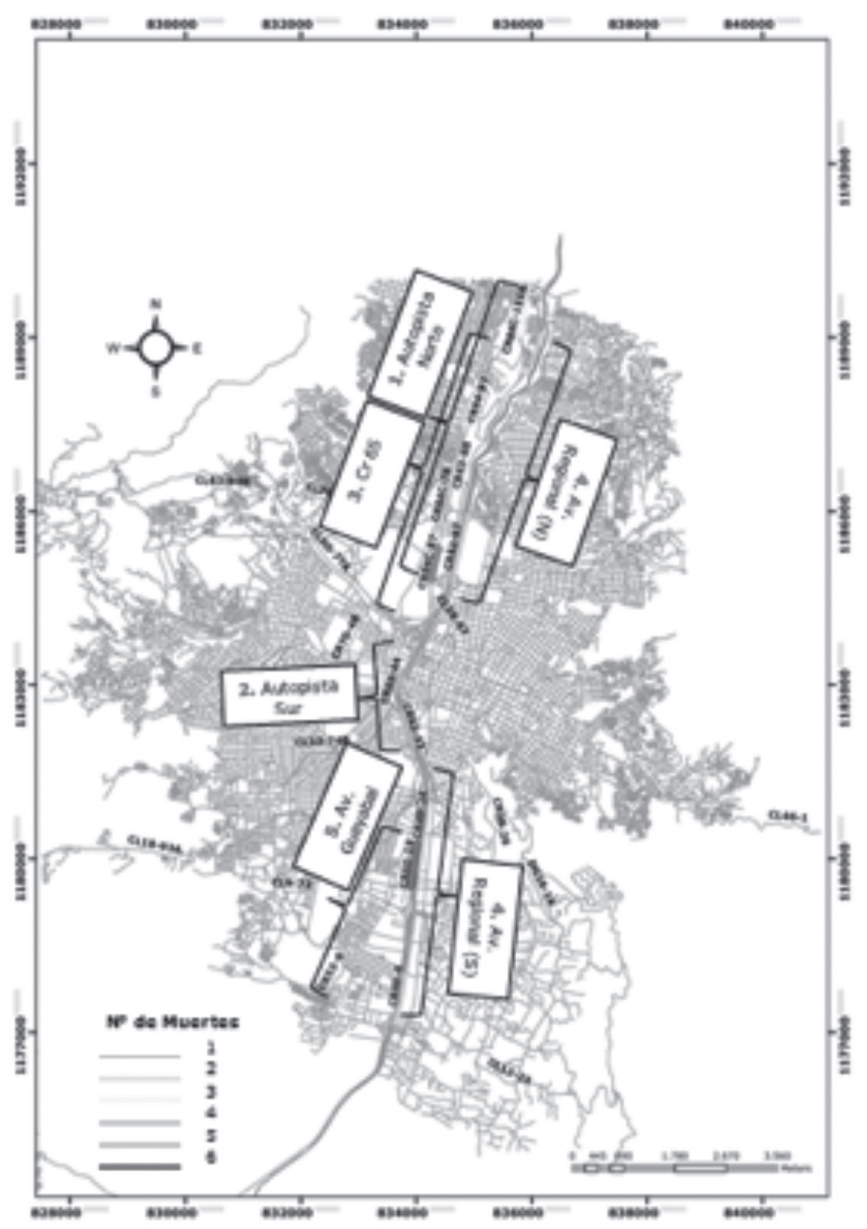

Figura 4. Distribución del número de motociclistas muertos en incidentes viales por la dirección de la ocurrencia. Medellín, 20092012

\section{Discusión}

La mortalidad ha sido explorada en diversos escenarios en las últimas décadas, porque su análisis constituye uno de los elementos fundamentales en el diseño y evaluación de políticas públicas; es un componente clave en la dinámica poblacional y es de gran utilidad para determinar las condiciones de salud de la población. Su estudio ha avanzado considerablemente hacia aquellas muertes que no deberían presentarse si las condiciones sociales, políticas y de los servicios de salud fueran diferentes, dado a que representan un indicador del avance e impacto de intervenciones en salud pública en un territorio [21].

En Medellín, durante el cuatrienio considerado los resultados revelaron que los usuarios de vehículos de motor de dos ruedas fueron la segunda población víctima de los incidentes viales que se presentaron en la ciudad, después de los peatones; la mayor tasa de mortalidad la presentaron los hombres en una relación de 5:1, siendo los motociclistas entre los 15 y 29 años de edad los más afectados, lo que conlleva a que la mayor pérdida de años potenciales de vida se haya concentrado en este mismo grupo etario tanto en los hombres como en las mujeres.

La tasa más alta de los APVP por los motociclistas se dio en el año 2011 con un incremento del 52,3\% con respecto al año anterior, lo cual constituye el cambio más notorio del periodo, dado a que en el año 2012 con respecto al inicial el aumento de la tasa de APVP fue de $10 \%$. La tendencia de las tasas de APVP seguida en los cuatro años 
revisados no es muy distante a la que se ha descrito en otra publicación donde el mayor número de años potenciales de vida perdidos que se concentró en los hombres jóvenes y adultos jóvenes de 15 a 39 años ha sido relacionado con la ocupación y la edad productiva, lo que representa un impacto económico desfavorable en términos de productividad social [14].

Según los resultados de otros estudios [12, 13, 22, 23], los motociclistas son la población que mayor riesgo tienen de presentar traumatismos fatales a causa de los eventos de tránsito, debido a que la mayoría de incidentes son choques contra objetos fijos, lo que ha representado un factor asociado significativamente con el trauma fatal de motociclistas, debido a que la energía que acumulan las víctimas en este tipo de accidentes es mayor que en otros [23].

En coherencia con los datos internacionales, nacionales y municipales sobre la mortalidad de los motociclistas, en los resultados de este trabajo, los hombres tuvieron protagonismo en la pérdida de los años potenciales de vida, casi el $80 \%$ con respecto al total, cifra que es similar al comportamiento descrito a nivel nacional, donde el 81,4\% de los APVP por accidentes de tránsito en el año 2010 tuvo a los hombres como protagonistas "[12] [14]; lo anterior ha sido relacionado con el hecho de que son los hombres quienes usan con mayor frecuencia las motocicletas como medio de transporte para cumplir con actividades cotidianas como trabajar, estudiar, recrearse o divertirse [10], y más cuando en Medellín, durante la última década se ha observado un cambio notorio en la movilidad vial, en la que se ha generalizado el uso de este vehículo como herramienta de transporte y de trabajo [14]; con un agravante, que no hay un control exhaustivo en la venta de estos vehículos que se percibe como factor de riesgo tanto para el usuario como para el peatón.

Otro de los resultados que más se destaca por su magnitud y su significación es la pérdida de años potenciales de vida por los motociclistas que se encuentran en el grupo etario de 15 a 49 años, con una concentración mayor en los jóvenes, dato que evidencia la pérdida de oportunidades en mano de obra productiva y reproductiva en Medellín por esta causa externa [24]; aunque la probabilidad de morir se incrementa con la edad, no se debe dejar de lado aquellas poblaciones que se consideran de menor riesgo, como lo son los jóvenes [25].

Una mención especial merece la pérdida de años potenciales de vida por los motociclistas que sufrieron incidentes viales en las zonas aledañas al río Medellín, donde el 60\% de los APVP en el periodo se concentraron en primer lugar en la zona noroccidental que se ha caracterizado por tener importantes vías de acceso a la ciudad como la autopista norte y la carrera 65, seguida de la zona Centro Oriental y Suroccidental; es así como las comunas en las que se observaron las mayores tasas de APVP fueron en su orden Castilla, La Candelaria, Guayabal y Aranjuez; aunque Aristizábal D, et, al. no calculó los APVP para dicha población, encontró que el trauma fatal de motociclistas se presentó con mayor frecuencia igualmente en estas comunas. De igual modo, en el anuario estadístico de accidentalidad vial de Colombia del año 2011, se encontró un comportamiento similar al antes descrito para ese año, donde a través de un mapa de riesgo para los accidentes graves ocurridos en Medellín se destacó la importante participación de las comunas Aranjuez, La Candelaria, Guayabal y el Poblado [23].

Es importante mencionar que los eventos fatales de los motociclistas en Medellín ocurrieron en su mayoría, en los tramos de algunas de las principales vías de la ciudad que se encuentran en los barrios Caribe, Guayabal, Castilla, Campo Amor y Cristo Rey donde las tasas de mortalidad y de APVP fueron mayores. En la autopista norte sobre la carrera $64 \mathrm{C}$ fue el tramo de la vía en que ocurrió el $5,2 \%$ de los hechos fatales de los motociclistas en Medellín, seguido de la autopista sur sobre la carrera 63 y la carrera 50, y en la carrera 65 ; estos resultados reiteran lo descrito en otras investigaciones afines a esta [23], que refieren que aunque en las glorietas e intersecciones se presentaban mayor número de accidentes viales, los motociclistas que conducían en vías diferentes a estas, por lo general tramos de vía, presentaban una probabilidad más alta de morir, lo cual se ha relacionado con el hecho de que son vías anchas que fomentan una mayor interacción entre los vehículos y la alta velocidad [26], que desde su concepción como vías principales permiten límites máximos, en el caso de Medellín, 80 kilómetros por hora, sumado a lo anterior, la inexperiencia de los motociclistas (27), el uso inadecuado de los cascos [28] y demás elementos de seguridad.

Como es de conocimiento, el sistema del tránsito es el más complejo y el que mayor peligro representa para las personas cotidianamente, debido a que es un fenómeno con múltiples interrelaciones que se dan dentro y fuera de él, donde factores como el aumento incontrolado en el número del parque automotor circulante, el crecimiento poblacional, la urbanización e industrialización inherentes al desarrollo económico y social de las poblaciones hacen que la capacidad infraestructural y controles emitidos para la regulación vial sean insuficientes para responder a las nuevas necesidades y retos; es por ello, que cada vez se hace necesario un trabajo interinstitucional coordinado, ya que como lo indica la OMS, la voluntad política y el compromiso de alcanzar una gestión eficaz de la seguridad, son dos puntos claves que permitirán lograr una pronta e importante reducción de los traumatismos causados por el tránsito [5].

Es fundamental que los planes, programas y proyectos encaminados a controlar la problemática vial desencadenada por los motociclistas en la ciudad estén orientados por sus necesidades específicas, para lo cual es indispensable la realización permanente de estudios que contribuyan con la comprensión de las situaciones que dan lugar a estos hechos que afectan principalmente a la población económicamente productiva; los hallazgos de estudios realizados a nivel mundial [28], sugieren que el uso del casco por estos usuarios de la vía reducen significativamente el riesgo de muerte en un accidente y por ende, la perdida de años potenciales de vida, por lo que se sugiere un control más riguroso en el uso de estos por parte de los agentes de tránsito; así mismo, el control en la entrega de licencias a motociclistas sin experiencia ha sido abordado en otros estudios [27] como un posible factor que influye en la mortalidad prematura de este grupo de riesgo, lo cual amerita una revisión más detallada para determinar el nivel de capacitación e inclusive la edad legal para conducir una motocicleta. Al igual que en otras ciudades, en Medellín se ha observado un crecimiento acelerado del parque automotor de motos en los últimos años; sin embargo, en la revisión bibliográfica no se encontró estudios que lo correlacionen con la accidentalidad en el país.

Es necesario llamar la atención sobre la frecuencia con que se presentan las lesiones fatales de los motociclistas en Medellín en la arteria vial aledaña al río que atraviesa la ciudad de sur a norte, ya que por lo general son vías importantes de comunicación con alto flujo vehicular, por lo que se recomienda revisar las condiciones y posibles factores desfavorables en la infraestructura y seguridad que presentan estos lugares para los motociclistas; así como la pertinencia de articular sistemas efectivos para controlar aquellas conductas que tienen un mayor riesgo de accidente, como el exceso de velocidad, lo cual se puede impulsar con la instalación de cámaras automáticas de velocidad o cinemómetros en aquellos sitios en los que se da la mayor accidentalidad de motociclistas, dado a que los accidentes producto de este exceso incrementa la severidad de las lesiones (Cendex). Además, se recomienda desarrollar continuamente trabajos que caractericen no sólo la mortalidad sino también la discapacidad generada en los usuarios que se encuentran en mayor riesgo en las vías de la ciudad.

En este estudio, es de resaltar que el subregistro en las muertes por causas externas en Medellín durante el periodo de estudio fue muy 
bajo, lo que corresponde con la afirmación del DANE, quien expone que el subregistro en la mortalidad y la omisión en la certificación por estas causas es mucho menor a la de las causas naturales, debido a que en su investigación intervienen varias instancias y el sistema de estadísticas vitales capta un número de dichas muertes que las autoridades competentes no llegan a conocer.

Por las características propias del diseño y la fuente de información, este trabajo presenta varias limitaciones: en primer lugar, la fuente de información que se empleó para el análisis no permitió hacer una descripción más detallada de la perdida de años potenciales de vida a causa de los incidentes viales de motociclistas por otras características importantes de persona, tiempo y lugar; debido a que el certificado de defunción ajustado por el DANE no registra a profundidad variables que describan la ocurrencia de los hechos, lo cual demuestra que en Colombia no hay coherencia entre las fuentes sanitarias y policiales de las causas externas, lo que se ha convertido en una debilidad institucional para consolidar las estadísticas oficiales [29].

Igualmente, la fuente de información no permitió identificar el lugar de la ocurrencia de los hechos para el 16\% de los registros de defunción de motociclistas en el cuatrienio; y aunque los conductores y pasajeros de dichos vehículos presentan niveles de riegos diferentes, no fue posible desagregar el análisis por cada uno de los usuarios debido a que la variable disponible para ello tendía a una descripción de "no especificado" en la condición de la víctimas, lo que implicaba que un número considerable de registros no se tuviesen en cuenta, de esta manera, se opta por trabajar de acuerdo con la clasificación que proporciona la CIE-10, donde los motociclistas se consideran bajo los códigos que van de la V20 a la V29 incluyendo así tanto a los conductores como a los pasajeros de motos.

Por otro lado, el método empleado para el cálculo de los años de vida perdidos por muerte prematura no permite comparar los resultados a nivel internacional, dado a que como límite de la edad para calcular los APVP se empleó la esperanza de vida al nacer que representa el perfil poblacional del país; sin embargo, en la revisión bibliográfica no se encontraron estudios en Colombia que detallaran los años potenciales de vida perdidos por la causa revisada específicamente en los motociclistas, lo cual fue una gran limitación para comparar los hallazgos de este trabajo. Y al ser un estudio descriptivo, no fue posible realizar análisis estadísticos que relacionaran los factores asociados y de causalidad con la pérdida prematura de años de vida por los motociclistas en la ciudad.

En conclusión, los resultados de este estudio son consistentes con la situación descrita en otras publicaciones. En Medellín los motociclistas jóvenes de sexo masculino presentan las tasas de APVP más altas del periodo, lo que implica mayores costos sociales y económicos para la ciudad ,y dado que la accidentalidad fatal más alta de este grupo de interés se presenta en las vías principales de la ciudad aledañas al río Medellín; es pertinente mencionar que aunque en los últimos años se han desarrollado importantes programas para atender a este sector poblacional, sus principales manifestaciones siguen estando aún presentes, al igual que muchos de los factores que lo desencadenan, por lo que el desafío consiste en que políticos, planificadores, promotores y, sobre todo los actores del sistema, tomen conciencia y se articulen para generar un espacio vial seguro.

\section{Conflicto de interés}

Los autores manifiestan no tener conflictos de interés

\section{Referencias}

1. Gómez H, Castro MV, Franco F, Bedregal P, Rodríguez García J, Espinoza A, et al. La carga de la enfermedad en países de América Latina. Salud pública Méx [Internet] 2011; 54. [Consultado 2014 May 20]. Disponible en: http://www.scielo.org.mx/scielo. php?pid=S0036-36342011000800003\&script=sci_arttext
2. Congreso de Colombia. Ley 1383 de 10 de marzo de 2010: por la cual se reforma la Ley 769 de 2002 - Código Nacional de Tránsito, y se dictan otras disposiciones. Bogotá: El Congreso; 2010.

3. Wong P, Salazar D, Bérninzon L, Rodríguez A, Salazar M, Valderrama $\mathrm{H}$, et al. Caracterización de los accidentes de tránsito en la región Callao-Perú, 1996-2004. Rev. peru. Epidemiol. [Internet] 2009; 13(3):1-7. [Consultado 2014 May 20]. Disponible en: http://rpe.epiredperu.net/rpe_ediciones/2009_v13_n03/ AO1_Vol13_No3_2009.html

4. González LM. Carga asociada a lesiones en términos de discapacidad y muerte como consecuencia de accidentes de tránsito en Colombia. [Trabajo de grado ingeniera industrial]. Bogotá: Pontificia Universidad Javeriana [Internet] 2011 [Consultado 2014 May 20]. Disponible en: http://repository. javeriana.edu.co/bitstream/10554/1673/1/Gonz\%C3\%A1lezBeltr anLuzMelba2011.pdf

5. Organización Mundial de la Salud. Informe sobre la Situación mundial de la seguridad vial 2009 [Internet]. [Consultado 2014 Abr 21]. Disponible en: http://www.who.int/violence_injury_ prevention/road_safety_status/2009/es/

6. Organización Mundial de la Salud. World reporto on road traffic injury prevention. Geneva 2004. [Internet]. [Consultado 2014 Abr 21]. Disponible en: http://www.who.int/violence_injury_ prevention/publications/road_traffic/world_report/es/

7. Organización Mundial de la Salud. Informe sobre la Situación mundial de la seguridad vial 2013 [Internet]. [Consultado 2014 Oct 20]. Disponible en: http://www.who.int/violence_injury_ prevention/road_safety_status/2013/report/es/

8. Comunidad Andina. Accidentes de tránsito en la comunidad Andina 2013. [Internet]. [Consultado 2014 Abr 21]. Disponible en: http://estadisticas.comunidadandina.org/eportal/ contenidos/2437_8.pdf

9. DANE. Encuesta nacional de calidad de vida 2010. [Internet] Bogotá: DANE; 2011. [Consultado 2014 Nov 28]. Disponible en: http://www.dane.gov.co/files/investigaciones/condiciones_vida/ calidad_vida/Boletin_Prensa_ECV_2010.pdf.

10. Londoño J. La carga de la enfermedad: un nuevo indicador en el campo de la salud pública. Rev. Fac. Nac. Salud Pública. 1996; 13(2):24-32.

11. Ospina C, Holguín H, Castañeda A, Marín D, Ballesteros M, Cadavid M. Capitulo estructura de la mortalidad. [Internet] 2010. [Consultado 2014 Nov 28]. Disponible en: http://www.dssa.gov. co/index.php/descargas/251-capituloestructuramortalidad/file

12. Cárdenas E. Diagnóstico sobre la accidentalidad de motociclistas en Medellín entre enero de 2004 y marzo de 2007. Rev Salud Pública de Medellín 2007;2(1):71-81

13. Alcaldía de Medellín. Informe anual de accidentalidad 2013 [Internet]. [Consultado 2014 Oct 23]. Disponible en: http:// www.medellin.gov.co/transito/archivos/accidentalidad/informe_ anual_2013.pdf

14. Vanegas Y, Cárdenas M. Años potencialmente perdidos por accidente de tránsito, Colombia 2010. Revista CES Salud Pública. 2011; 2(2):159-168.

15. Organización Panamericana de la Salud. Técnicas para la medición del impacto de la mortalidad: Años Potenciales de Vida Perdidos. [Internet]. [Consultado 2014 Nov 29]. Disponible en: http://www1.paho.org/spanish/dd/ais/be_v24n2-APVP.htm

16. DANE. Estudios postcensales. Estimación de la mortalidad 1985-2005. Bogotá D.C., Colombia [Internet] 2009. [Consultado 2014 Nov 3].Disponible en: https://www.dane.gov.co/files/ investigaciones/poblacion/proyepobla06_20/7Proyecciones_ poblacion.pdf 
17. Organización Panamericana de la Salud. Sobre la estimación de tasas de mortalidad para países de la Región de las Américas. Bol Epidemiol, [Internet] 2003; 24 (4):1-16. [Consultado 2014 Nov 3]. Disponible en: http://cidbimena.desastres.hn/docum/ops/ publicaciones/Epidemiologico/EB_v24n4.pdf

18. ArcGIS. Clasificar campos numéricos para simbología graduada [Internet]. [Consultado 2015 Feb 6]. Disponible en: http://help.arcgis.com/es/arcgisdesktop/10.0/help/index. html\#//00s50000001r000000

19. Vargas JA, Horfan D. Proceso de geocodificación de direcciones en la ciudad de Medellín, una técnica deterministica de georreferenciación de direcciones. Ing. USBMed [Internet] 2013; 4 (1): 6-21. [Consultado 2015 Ene10]. Disponible en: http://web. usbmed.edu.co/usbmed/fing/v4n1/v4n1al.pdf

20. Gobierno de Chile. Comisión Nacional de Seguridad de Tránsito. Análisis de la distribución espacial de los accidentes de tránsito y sus víctimas en el Gran Santiago, Diagnostico 2010. [Internet]. [Consultado 2015 Feb 7]. Disponible en: http://paritario.utalca. cl/docs/MESA_B_PONENCIAS/B_19_Accidentes_Transito_ Santiago_Romanette_Soto.pdf

21. López A. Evolución de la estructura de las muertes evitables en la frontera norte de México entre 1998 y 2007 y sus factores condicionantes [Tesis Maestra en Demografía]. México: El Colegio de la Frontera Norte; 2010.

22. Comisión Económica para América Latina y el Caribe. Seguridad vial y salud pública: costos de atención y rehabilitación de heridos en Chile, Colombia y Perú [Internet]. [Consultado 2014 Nov 11]. Disponible en: http://www.cepal.org/transporte/noticias/ bolfall/7/48857/FAL-311-WEB.pdf

23. Aristizábal D, González G, Suárez J F, Roldán P. Factores asociados al trauma fatal en motociclistas en Medellín, 20052008. Biomédica. 2012; 32(2): 3-37.

24. Cardona A, Segura C, Ángela M, Berbesí F, Dedsy Y. Mortalidad y años de esperanza de vida perdidos en cinco zonas de Medellín, 2004-2006. Rev. Fac. Nac. Salud Pública. 2011; 29(2), 189-198.

25. Grisales H, Caicedo B, Serna C, Uribe D. Causas de mortalidad en jóvenes y su contribución al cambio en la esperanza de vida: Cali, 1989-1991. Colomb. Med. [Internet] 2005; 36(2): 85-93. [Consultado 2015 Feb 17]. Disponible en: http://www.scielo. org.co/scielo.php?pid=S1657-95342005000200005\&script $=$ sci_ arttext\#1

26. Jimenez A, Bocarejo J P, Zarama R, Yerpez J. A case study analysis to examine motorcycle crashes in Bogota, Colombia. Journal of Safety Research. 2015. [Internet]. [Consultado 2015 Feb 10]. Disponible en: http://www.ncbi.nlm.nih.gov/pubmed/25662880

27. Kardamanidis K, Martiniuk A, Ivers RQ, Stevenson MR, Thistlethwaite K. Motorcycle rider training for the prevention of road traffic crashes. Cochrane Database Syst Rev. [Internet] 2010. [Consultado 2015 Feb 17]. Disponible en: http://www.ncbi.nlm. nih.gov/pubmed/20927741

28. Liu BC, Ivers R, Norton R, Boufous S, golpes S, Lo SK. Cascos para la prevención de lesiones en motociclistas. La Biblioteca Cochrane. [Internet] 2008. [Consultado 2015 Feb 17]. Disponible en: http://www.cochrane.org/es/CD004333/cascos-para-laprevencion-de-lesiones-en-motociclistas

29. Alcaldía de Medellín. Así se vivió en Medellín el VI Foro Internacional de Movilidad [Internet] Medellín: La Alcaldía; 2014 [acceso 20 de febrero de 2015]. Disponible en: https:// www.medellin.gov.co/movilidad/component/k2/asi-se-vivio-enmedellin-el-vi-foro-internacional-de-movilidad 